\title{
THE THIRD HARMONIC IN SOLAR RADIO BURSTS
}

\author{
V. V. ZHELEZNYAKOV and E. Ya. ZLOTNIK \\ Radiophysical Research Institute, Gorkii, U.S.S.R.
}

(Presented by D. B. Melrose)

\begin{abstract}
Generation of third harmonic plasma emission by coalescence of second harmonic radiation with a plasma wave is more favourable than by direct coalescence of three plasma waves. The predicted ratio of the intensities at the third (III) and second (II) harmonics is $I_{\omega}^{\mathrm{III}} / I_{\omega}^{\mathrm{II}} \sim \omega_{L} W_{l} L / N m c^{3}$, where $\omega_{L}$ is the plasma frequency, $W_{l}$ is the energy density in plasma waves, $L$ the linear dimensions of the source and $N$ the ambient electron number density. The intensity at the second harmonic is

$$
I_{\omega}^{\mathrm{II}} \simeq e^{2} W_{l}^{2} L_{N} / m^{2} c^{2} \omega_{L}^{2},
$$

where $L_{N}\left(\sim 10^{10} \mathrm{~cm}\right.$ at $\left.\omega_{L} \sim 2 \pi \times 10^{8} \mathrm{~s}^{-1}\right)$ is the typical dimension of coronal inhomogeneities.

It is suggested that the third harmonic in a $U$-burst observed by Haddock and Takakura and in type $\mathrm{V}$ emission reported by Benz (1973) were due to this process. For the latter event the theory predicts $I^{\mathrm{III}} / I^{\mathrm{II}} \sim 3 \times 10^{-2}$ compared with the observed ratio of $10^{-1}$.
\end{abstract}




\section{DISCUSSION}

\section{(After Two Abstracts of Zaitsev et al. and Zheleznyakov and Zlotnik)}

Rosenberg: (1) Continuous injection seems difficult to reconcile with X-ray data. Is that true? (2) The fall-off with $E^{-6}$ or $E^{-3}$ seems to contradict the observed exponential decay. (3) Has anybody observed third harmonics? In my opinion the two cases mentioned should be used with care.

Melrose: As I understand it, continuous injection would give type III burst the same duration at all frequencies up to the $30 \mathrm{MHz}$ the authors mention. The power law decay is considered satisfactory by the authors.

Smith: There is nothing difficult about a short continuous injection of a few tenths of a second. Indeed, physically the injection time must be finite. Zaitsev et al. appear to obtain agreement with the data on decay because they considered a limited frequency range and drew sufficiently large dots around the data points.

Takakura: Zaitsev et al. have neglected the induced scattering of plasma waves in the resonance region into the non-resonance region. However, if we adopt their solution and take the observed flux of solar electrons at the Earth, the time scale for induced scattering is less than the growth time of the plasma waves in the resonance region everywhere between the Sun and the Earth. Therefore their result is inconsistent. I have modified their result taking into account induced scattering, but there is no time to go into the details.

Smith: A problem with the induced scattering time of Tsytovich is that it needs to be multiplied by a factor of about 600 for a narrow spectrum of waves according to the numerical analysis which I carried out with Fung and by an even larger factor for a broad spectrum of waves. With this correction, induced scattering would not lead to a significant change in the angular distribution of waves for a broad spectrum of waves.

Melrose: The fact that fundamental radiation must be amplified to obtain observed brightness temperatures requires that induced scattering of plasma waves also be important since the process is the same for both waves.

Wild: We have looked for a third harmonic for many years. It often looks possible, but there is no convincing case.

Kane: There is evidence for continuous acceleration or injection of electrons in the observed hard $\mathrm{X}$-ray emission and its time variation. This is independent of the model assumed, i.e. $\mathrm{a}$ thin or thick target model for the X-ray source. 